\section{Vibrational Frequencies of Molecules}

IN a recent letter to NATURE $^{1}$, I submitted evidence that the ground state vibrational frequency of a molecule $A B$ was the mean of the frequencies of the molecules $A_{2}$ and $B_{2}$ provided that $A$ and $B$ belong to the same group of the Periodic Table. I was then unaware of the work of Dr. C. H. Douglas Clark ${ }^{2}$, who had previously arrived at the same conclusion. To account for certain deviations from this rule, he adds the further condition that the frequencies of $A_{2}$ and $B_{2}$ must not be too dissimilar in magnitude.

If the motion of a diatomic molecule $A B$ be considered simple harmonic, the vibrational frequency $\omega$ is given by the expression:

$$
\omega=\frac{1}{2 \pi}-\sqrt{\frac{M_{A}+M_{B}}{M_{A} \cdot M_{B}} \cdot K},
$$

where $M_{A}$ and $M_{B}$ are the atomic weights of $A$ and $B$ respectively and $K$ is the force constant. It is reasonable to assume in the first instance that for such molecules as $A_{2}, B_{2}$ and $A B$, the force constant $K$ will have the same value. A simple calculation then shows that the above relation between the vibrational frequencies follows if the masses $M_{A}$ and $M_{B}$ are of the same order. Consequently the frequencies of $A_{2}$ and $B_{2}$ should also be of the same order, thus accounting for Clark's second condition.

H. G. Howell.

Armstrong College,

Newcastle-on-Tyne.

${ }^{1}$ H. G. Howell, Nature, 138, 36 (1936).

${ }^{2}$ C. H. Douglas Clark, Trans. Faraday Soc., 31, 1017 (1935).

\section{Asymmetry in Metallic Zinc and Cadmium}

In the issue of Nature of February 22, Dr. G. W. Brindley reports on discrepancies in the $\mathrm{X}$-ray scattering factors of zinc and cadmium. The observed values do not lie on a smooth curve. Of the two possible interpretations of this phenomenon, namely $(a)$ the asymmetry of the atom or $(b)$ the asymmetry in the lattice vibrations, Dr. Brindley gives preference to the latter, since the deviations are too large to be explained by $(a)^{1}$.

Nevertheless, I wish to say that the possibility of an appreciable variation from a spherical shape of the zinc and cadmium atoms is not altogether out of the question. This opinion is based on the two following observations :

(l) Nineteen of the twenty-one elements having the close-packed hexagonal structure ${ }^{2}$ show a ratio $c / a$ between $1 \cdot 578_{5}(\mathrm{Os})$ and $1 \cdot 64(\mathrm{Ni})$. This implies variations of only $-3 \cdot 3$ per cent and +0.43 per cent respectively from the value $\mathrm{I} \cdot 633$, which corresponds to the case of close-packed spheres. Cadmium and zine, however, show values of $\mathrm{I} \cdot 885_{9}$ and $1 \cdot 856_{2}$ $\left(1 \cdot 884_{2}\right.$ resp, at $\left.415^{\circ}\right)$, these ratios being $15 \frac{1}{2}$ per cent higher. This difference seems to be too large to be explained by thermal oscillations, should this be taken as the reason for the deviations in the case of the other metals.

(2) The following calculation seems to be of still greater weight. In pursuance of the hypothesis that cadmium and zinc atoms are rotational ellipsoids ${ }^{3}$, the question arises, what would be the shape of the ellipse forming the cross-section, that is, what would be its numerical eccentricity. The answer is given by the following calculation for cadmium ( $p=$ major, $q=$ minor axis) :

$$
\frac{p}{q}=\frac{1}{\sqrt{1-\varepsilon^{2}}}=\frac{(c / a)_{\text {ellipse }}}{(c / a)_{\text {sphere }}}=\frac{1 \cdot 885_{9}}{1 \cdot 6333},
$$

and hence $\varepsilon=0.5002$; that is, equal to $1 / 2$ as precisely as possible*. It is difficult to believe that this is a mere chance, especially in view of the considerations mentioned under (1). (There is no intermediate value between $1 \cdot 64$ and $1 \cdot 856_{2}$ ).

The foci of this ellipse are situated at the centre of each half of its major axis. It is therefore possible to put a sphere in each half of the rotational ellipsoid, their centres coinciding with the foci and their surfaces touching the vertices and the centre of the ellipsoid. Such a rotational ellipsoid, being a 'quasi double-sphere', can be regarded as a body very nearly related to the sphere as to its degree of symmetry.

\section{Technische Hochschule,}

K. HerrmanN. Berlin.

¿ See also the paper by C. Zener, Phys. Rev., 49, 122 (1936) 2 See M. C. Neuburger, "Gitterkonstanten 1936", Z. Krist., 93, 1 (1936).

${ }^{3}$ This view has been mentioned by various writers: Hull, Ewald Hume-Rothery. See also Canfleld, Phys. Rev., 35, 530 (1930),

4 For zinc at room-temperature with $1.856_{2}$, one gets $\varepsilon=0 \cdot 475_{6}$ and at $415^{\circ}$ with $1 \cdot 884_{2}, \varepsilon=0 \cdot 4989$.

Through the kindness of the Editor, I have been allowed to see Prof. Herrmann's letter prior to its publication. I entirely agree that the $\mathrm{X}$-ray results do not exclude the possibility that the outermost electrons of the atoms in metallic zinc and cadmium may depart from having spherical symmetry; one would not expect the completed inner electron shells to have appreciable asymmetry. The possibility of the outermost electrons being asymmetrical, however, is not to be regarded as an alternative explanation of the experimental results, but as something additional to the explanation already given in terms of asymmetrical lattice vibrations. The contributions of the outermost electrons to the scattering factors of zinc and cadmium at all angles for which measurements can be made are so small that one could scarcely hope to measure an asymmetry in their distribution by means of X-rays. On the other hand, any asymmetry in the lattice vibrations will affect the entire atom, and at large scattering angles, measurable effects may be expected if the asymmetry is sufficiently great, and this appears to be so for zinc and cadmium.

With regard to point (1) raised by Prof. Herrmann, it is difficult to see whether this indicates an asym. metry of the atoms or of the lattice vibrations, with. out going into the matter much more fully. If, how ever, the unusually high $c / a$ values of zinc and cadmium are associated with strongly asymmetrical lattice vibrations, then one might expect that a 'normal' metal, such as magnesium with $c / a=1 \cdot 624$, would not show strongly asymmetrical vibrations. I have recently carried out an $\mathrm{X}$-ray investigation of magnesium similar to the previous experiments on zinc and cadmium, and the results so far obtained suggest that in magnesium the lattice vibrations are very much more nearly isotropic than in zinc and cadmium. These results appear to be consistent with the fact that, whereas in zine and cadmium the thermal expansion coefficients perpendicular and 